\title{
Differentiation between polycystic ovary syndrome and polycystic ovarian morphology by means of an anti-Müllerian hormone cutoff value
}

\author{
Do Kyeong Song, Jee-Young Oh, Hyejin Lee, and Yeon-Ah Sung
}

Department of Internal Medicine, Ewha Womans University School of Medicine, Seoul, Korea

\author{
Received: February 9, 2016 \\ Revised : May 22, 2016 \\ Accepted: May 29, 2016

\section{Correspondence to} \\ Do Kyeong Song, M.D. \\ Department of Internal Medi- \\ cine, Ewha Womans University \\ School of Medicine, 1071 Anyang- \\ cheon-ro, Yangcheon-gu, Seoul \\ 07985 , Korea \\ Tel: +82-2-2650-6158 \\ Fax: +82-2-2650-5235 \\ E-mail: sdkhope@hanmail.net
}

Background/Aims: Although increased serum anti-Müllerian hormone (AMH) level has been suggested to be a surrogate marker of polycystic ovarian morphology (PCOM), its association with polycystic ovary syndrome (PCOS) is controversial, and its diagnostic value has not been determined. We aimed to observe the relationship between the AMH level and PCOS phenotypes and to determine the optimal cutoff value of AMH for the diagnosis of PCOS in young Korean women. Methods: We recruited 207 women with PCOS (120 with PCOM and 87 without PCOM) and 220 regular cycling women with normoandrogenemia (100 with PCOM and 120 without PCOM). Subjects underwent testing at a single outpatient visit. Serum AMH level was measured.

Results: Women with PCOS had higher serum AMH levels than did regular cycling women with normoandrogenemia $(p<0.05)$. Women with PCOM had higher serum AMH levels than women without PCOM, regardless of PCOS status ( $p$ $<0.05$ ). The optimal AMH cutoff value for the diagnosis of PCOS was $10.0 \mathrm{ng} / \mathrm{mL}$ (71\% sensitivity, 93\% specificity). Serum AMH was an independent determinant of total testosterone after adjustment for age, body mass index, and the number of menses/year $(\beta=0.31, p<0.01)$. An association between AMH and hyperandrogenism was only observed in women with PCOS, and it was independent of the presence of PCOM.

Conclusion: The serum AMH level can be useful for the diagnosis of PCOS at any age less than 40 years, and the optimal cutoff value for the diagnosis of PCOS identified in this study of young Korean women was $10.0 \mathrm{ng} / \mathrm{mL}$.

Keywords: Anti-Mullerian hormone; Polycystic ovary syndrome; Ovarian cysts

\section{INTRODUCTION}

Polycystic ovary syndrome (PCOS), which is the most common endocrine disorder in reproductive-aged women, affects $6 \%$ to $10 \%$ of premenopausal women [1]. The diagnosis of PCOS is based on a combination of clinical, biochemical, and ultrasound criteria, and the main diagnostic criteria of PCOS are polycystic ovarian mor- phology (PCOM), oligo-anovulation, and hyperandrogenism (HA) [2]. However, the diagnosis of PCOS can be subjective, because counting the ovarian follicular number and measuring the ovarian volume using ovarian ultrasound are technique-dependent and because obtaining standardized measurements is difficult. The number of follicles and the size of the ovary measured by ovarian ultrasound may overlap in women with and 
without PCOS, and PCOM has been reported frequently in women without PCOS [3]. Moreover, there is currently no consensus on the most appropriate androgen to measure for PCOS diagnosis, and diverse androgen cutoff values have been suggested for the determination of biochemical hyperandrogenemia and diagnosis of PCOS because direct immunoassay methods can be highly inaccurate for the measurement of testosterone when its level is at the lower end of the female range [4].

Anti-Müllerian hormone (AMH) is secreted by granulosa cells and small antral and preantral follicles [5]. Serum AMH levels were markedly increased in women with PCOS and were positively related with small antral follicle number $[6,7]$. The AMH level has been reported to be independently related to HA and PCOM [8] and to be strongly associated with the main phenotypic features of PCOS, including ovulatory dysfunction [9,10]. Therefore, increased serum AMH level has been suggested as an objective surrogate marker for the diagnosis of PCOS and has been shown to be correlated with conventional diagnostic criteria [9,11].

It is difficult to determine the optimal AMH cutoff value for the diagnosis of PCOS because the age-related decline in antral follicle number is reflected by AMH concentration. A variety of AMH cutoff values for predicting PCOS have been proposed in previous studies $[12,13]$. In the same age group, the serum AMH level was higher in PCOS women than in those without PCOS, and age-specific AMH cutoff values for Chinese women with and without PCOS have been suggested [14]. In addition, an AMH Z-score, the level of AMH relative to the expected mean and standard deviation for a particular age, was proposed to adjust for the age-related decline in the number of antral follicles [15]. The aim of this study was to observe the relationship between the AMH level and PCOS phenotypes and to determine the optimal AMH cutoff value for the diagnosis of PCOS in young women.

\section{METHODS}

\section{Subjects}

Between 2008 and 2010, we performed a survey of the menstrual health of young women under 40 years of age living in Seoul, Korea. Participants were recruited us- ing newspaper and online advertisements. We enrolled 207 women with PCOS and 220 regular cycling women with normoandrogenemia. As proposed at the European Society for Human Reproduction and Embryology (ESHRE) [2], PCOS was diagnosed when two or more of the following three criteria were met: oligo- or anovulation, $\mathrm{HA}$, and polycystic ovaries. Oligomenorrhea (OM) was defined as having fewer than eight menstrual cycles per year. Biochemical hyperandrogenemia was defined as a total testosterone level or free testosterone level above the 95th percentile (total testosterone $\geq 67$ $\mathrm{ng} / \mathrm{dL}$ or free testosterone $\geq 0.84 \mathrm{ng} / \mathrm{dL}$ ) for testosterone levels in healthy women with regular menstrual cycles [16]. Clinical HA was defined as hirsutism with a modified Ferriman-Gallwey score of $\geq 3$ [17]. HA included biochemical hyperandrogenemia and/or clinical HA. One of the following two criteria must be met for the diagnosis of PCOM: visualization of $\geq 12$ follicles/ovary that measure 2 to $9 \mathrm{~mm}$ in diameter or an ovarian volume of $>10 \mathrm{~cm}^{3}$ identified by transvaginal ultrasonography or transrectal ultrasonography with a distended bladder for virginal women. Patients with similar clinical presentations, such as congenital adrenal hyperplasia, androgen-secreting tumors, and Cushing's syndrome, were excluded from the study. Patients with 21-hydroxylase-deficient nonclassic adrenal hyperplasia were excluded based on the basal morning level of 17 -hydroxyprogesterone. Subjects were excluded if they had been on medication (e.g., steroids, oral contraceptives, metformin, or thiazide diuretics) within 3 months of the evaluation or had used other drugs that could affect basal parameter status. Subjects were excluded if they had menstrual irregularities or clinical signs of HA compared to regular cycling women with normoandrogenemia. We divided the subjects based on the presence of PCOS and PCOM. Among the women with PCOS, 120 had PCOM (group 1, HA + OM + $\mathrm{PCOM}$ ) and 87 did not have PCOM (group 2, HA + OM). Among the regular cycling women with normoandrogenemia, 100 had PCOM (group 3, PCOM), and 120 did not have PCOM (group 4, control). The subjects were divided into five groups according to age: $<20,20$ to 24,25 to 29,30 to 34 , and $\geq 35$ years. Written informed consent was obtained from all participants, and the Institutional Review Board of Ewha Womans University Mokdong Hospital approved this study. 
Table 1. Clinical, biochemical, and ultrasound data of subjects

\begin{tabular}{|c|c|c|c|c|}
\hline Variable & Group $1(\mathrm{HA}+\mathrm{OM}+\mathrm{PCOM})$ & Group 2 (HA + OM) & Group 3 (PCOM) & Group 4 (control) \\
\hline Subjects no. & 120 & 87 & 100 & 120 \\
\hline Age, yr & $25 \cdot 0 \pm 4 \cdot 7$ & $23.9 \pm 4.6$ & $25 \cdot 5 \pm 4.0$ & $24.8 \pm 4.6$ \\
\hline Body mass index, $\mathrm{kg} / \mathrm{m}^{2}$ & $23.0 \pm 3.7$ & $23.0 \pm 4.8$ & $22.4 \pm 3.2$ & $22.9 \pm 2.7$ \\
\hline Waist circumference, $\mathrm{cm}$ & $77.3 \pm 9.6$ & $76.5 \pm 10.4$ & $74 \cdot 5 \pm 8.6$ & $76.0 \pm 6.5$ \\
\hline Total testosterone, $\mathrm{nmol} / \mathrm{L}$ & $2.5 \pm 0.6^{\mathrm{a}, \mathrm{b}, \mathrm{c}}$ & $2.3 \pm 0.6^{\mathrm{a}, \mathrm{b}}$ & $1.5 \pm 0.4$ & $1.5 \pm 0.5$ \\
\hline $\mathrm{SHBG}, \mathrm{nmol} / \mathrm{L}$ & $56.5 \pm 30.8^{\mathrm{a}, \mathrm{b}}$ & $55.8 \pm 37.4^{\mathrm{a}, \mathrm{b}}$ & $107.2 \pm 60.5$ & $96.5 \pm 49.0$ \\
\hline Free testosterone, nmol/L & $0.03 \pm 0.01^{\mathrm{a}, \mathrm{b}}$ & $0.03 \pm 0.01^{\mathrm{a}, \mathrm{b}}$ & $0.01 \pm 0.01$ & $0.01 \pm 0.01$ \\
\hline $\mathrm{AMH}, \mathrm{ng} / \mathrm{mL}^{\mathrm{d}}$ & $18.6(13.5-21.0)^{\mathrm{a}, \mathrm{b}, \mathrm{c}}$ & $9.1(7.0-13.0)^{\mathrm{a}}$ & $10.3(8.0-14.6)^{\mathrm{a}}$ & $5 \cdot 7(4.2-7.9)$ \\
\hline Ovarian volume, $\mathrm{cm}^{3}$ & $10.7 \pm 3.7^{\mathrm{a}, \mathrm{b}, \mathrm{c}}$ & $5 \cdot 3 \pm 1.6^{b}$ & $8.4 \pm 3.1^{\mathrm{a}}$ & $4.8 \pm 1.7^{b}$ \\
\hline Follicle no. & $14.2 \pm 6.4^{\mathrm{a}, \mathrm{b}, \mathrm{c}}$ & $6.8 \pm 2.3^{b}$ & $10.8 \pm 2.0^{a}$ & $6.4 \pm 2.0^{b}$ \\
\hline No. of menses/yr & $5 \cdot 5 \pm 2.0^{a, b}$ & $5 \cdot 3 \pm 1.6^{\mathrm{a}, \mathrm{b}}$ & $10.0 \pm 0.0$ & $10.0 \pm 0.0$ \\
\hline
\end{tabular}

Values are presented as mean $\pm \mathrm{SD}$ or median (interquartile range).

HA, hyperandrogenism; OM, oligomenorrhea; PCOM, polycystic ovarian morphology; SHBG, sex hormone-binding globulin; AMH, anti-Müllerian hormone.

${ }^{a} p<0.05$ vs. group 4 .

b $p<0.05$ vs. group 3.

${ }^{c} p<0.05$ vs. group 2.

${ }^{\mathrm{d} B y}$ one-way Kruskal-Wallis variance analysis with post hoc pair-wise comparisons with Bonferroni's corrected Mann-Whitney U test.

\section{Methods}

Weight and height were measured with the subjects wearing light clothing and no shoes; body mass index (BMI) was also calculated $\left(\mathrm{kg} / \mathrm{m}^{2}\right)$. Waist circumference was measured to the nearest $0.1 \mathrm{~cm}$ on bare skin during mid-respiration at the narrowest indentation between the 1oth rib and the iliac crest.

On the third day of the follicular phase of the menstrual cycle, a venous blood sample was obtained from each subject after an overnight fast of at least 8 hours. In women with amenorrhea, the blood samples were obtained on a random day. Total testosterone levels were measured via the chemiluminescent immunoassay method using a commercially available kit (Siemens, New York, NY, USA, mean inter- and intra-assay coefficient of variation (CV) of $4.4 \%$ and $6.2 \%$, respectively). Sex hormone-binding globulin (SHBG) levels were measured by immunoradiometric assay using a commercially available kit (DPC, Los Angeles, CA, USA, mean inter- and intra-assay CVs of 7.9\% and 5.3\%, respectively). Free testosterone levels were calculated using the formula available on the International Society for Study of the Aging Male (ISSAM) web site, which is based on total testosterone, SHBG, and albumin levels obtained in the same sample from each subject. Serum AMH level was measured by enzyme-linked immunosorbent assay using a commercially available kit (Beckman Coulter, Chaska, MN, USA, mean inter- and intra-assay CVs of $4.5 \%$ and $3.6 \%$, respectively). Serum AMH levels were compared between the five age groups.

Ultrasound examinations were performed with a 7 $\mathrm{MHz}$ transvaginal (or transrectal for virginal women) transducer (Logic 400 General Electric, GE Medical Systems, Milwaukee, WI, USA). Ovarian volume was calculated according to a simplified formula for an ellipsoid (0.5 $\times$ length $\times$ width $\times$ thickness) [18]. Ovarian volume was defined as the average volume of both ovaries, and the ovarian follicle number was defined as the average number of follicles in each ovary.

\section{Statistical analysis}

The statistical analyses were performed using the SPSS version 18.o (SPSS Inc., Chicago, IL, USA). The Kolmogorov-Smirnov statistic was used to analyze continuous variables for normality. Quantitative variables were reported as the mean \pm standard deviation. Variables with skewed deviations were reported as medians and interquartile ranges. Group mean values were compared us- 
Table 2. Partial correlation analysis between anti-Müllerian hormone levels and the various parameters after adjustment for age

\begin{tabular}{|c|c|c|c|c|}
\hline \multirow{2}{*}{ Parameter } & \multicolumn{2}{|c|}{ Women with PCOS (group 1, 2) } & \multicolumn{2}{|c|}{ Women without PCOS (group 3, 4) } \\
\hline & $r$ & $p$ value & $r$ & $p$ value \\
\hline Body mass index & -0.08 & 0.23 & -0.06 & 0.40 \\
\hline Waist circumference & -0.07 & 0.31 & -0.07 & 0.34 \\
\hline Total testosterone & 0.33 & $<0.01$ & 0.06 & 0.35 \\
\hline SHBG & -0.03 & 0.71 & -0.07 & 0.35 \\
\hline Free testosterone & 0.16 & 0.02 & 0.07 & 0.34 \\
\hline Ovarian volume & 0.57 & $<0.01$ & 0.24 & $<0.01$ \\
\hline Follicle no. & 0.39 & $<0.01$ & 0.47 & $<0.01$ \\
\hline No. of menses/yr & -0.09 & 0.22 & - & - \\
\hline
\end{tabular}

PCOS, polycystic ovary syndrome; SHBG, sex hormone-binding globulin.

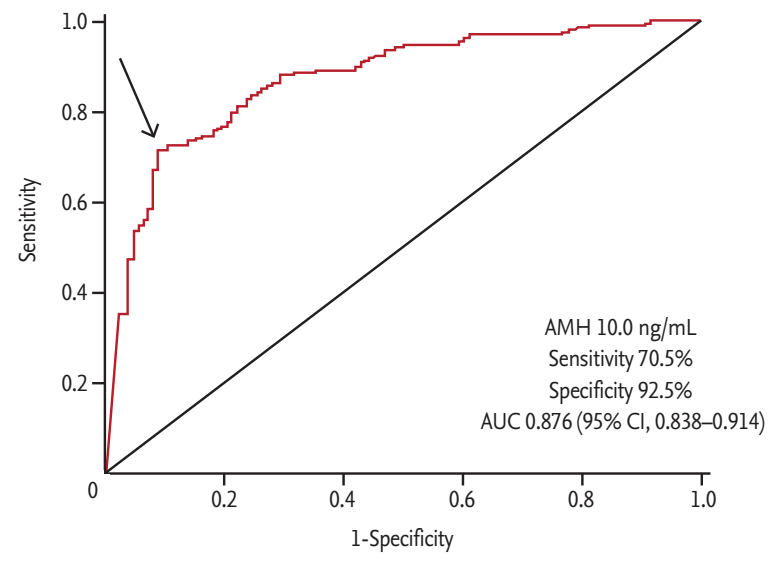

Figure 1. Receiver-operating characteristic curve for the diagnostic capability of serum anti-Müllerian hormone (AMH) levels for diagnosis of polycystic ovary syndrome. AUC, area under the curve; CI, confidence interval.

ing one-way analysis of variance. All other data were evaluated by one-way Kruskal-Wallis variance analysis with post hoc pair-wise comparisons using Bonferroni's corrected Mann-Whitney $U$ test. The age-adjusted partial correlation analysis was used to determine the relationships between AMH level and the various parameters. Multiple linear regression analyses were performed to determine the independent association between AMH levels and total testosterone after controlling for age, BMI, and the number of menses/year. A multiple logistic regression analysis was used to assess the association between AMH levels and the presence of PCOS. A receiver-operating characteristic curve was constructed to evaluate the sensitivity and specific- ity of AMH level for the prediction of PCOS. A $p<0.05$ was considered statistically significant.

\section{RESULTS}

The clinical, biochemical, and ultrasound data of the four groups (group 1, HA + OM + PCOM; group 2, HA + OM; group 3, PCOM; and group 4, control) are summarized in Table 1. Serum AMH level was the highest in group 1, intermediate in groups 2 and 3, and lowest in group 4. Serum AMH in group 1 was significantly higher than in the three other groups (all $p<0.05$ ). The serum AMH levels in group 2 and group 3 were similar to each other but were significantly higher than that of group $4(p<0.05)$. The values of age, BMI, and waist circumference did not differ significantly among the four groups (all $p>0.05$ ). Women with PCOS had higher values of total testosterone and free testosterone and lower values of SHBG and number of menses/year than did regular cycling women (all $p<0.05$ ). The values of ovarian volume and follicle number were highest in group 1, intermediate in group 3 , and lowest in groups 2 and 4 (all $p<0.05)$.

The serum AMH level was positively correlated with total testosterone $(r=0.33)$, free testosterone $(r=0.16)$, ovarian volume $(r=0.57)$, and follicle number $(r=0.39)$ after adjustment for age in women with PCOS (groups 1 and 2 , all $p<0.05$ ). In regular cycling women without PCOS, serum AMH level was also positively correlated with ovarian volume $(r=0.24)$ and follicle number $(r=$ 
Table 3. Partial correlation analysis between anti-Müllerian hormone levels and the various parameters after adjustment for age

\begin{tabular}{|c|c|c|c|c|c|c|c|c|}
\hline \multirow{2}{*}{ Parameter } & \multicolumn{2}{|c|}{ Group $1(\mathrm{HA}+\mathrm{OM}+\mathrm{PCOM})$} & \multicolumn{2}{|c|}{ Group $2(\mathrm{HA}+\mathrm{OM})$} & \multicolumn{2}{|c|}{ Group 3 (PCOM) } & \multicolumn{2}{|c|}{ Group 4 (control) } \\
\hline & $r$ & $p$ value & $r$ & $p$ value & $r$ & $p$ value & $r$ & $p$ value \\
\hline $\mathrm{BMI}$ & -0.002 & 0.986 & -0.175 & 0.106 & -0.013 & 0.898 & -0.026 & 0.776 \\
\hline WC & -0.037 & 0.691 & -0.176 & 0.104 & -0.025 & 0.803 & -0.003 & 0.973 \\
\hline Total testosterone & 0.208 & 0.023 & 0.347 & 0.001 & 0.003 & 0.980 & 0.171 & 0.063 \\
\hline SHBG & -0.076 & 0.411 & 0.042 & 0.704 & -0.193 & 0.089 & -0.006 & 0.954 \\
\hline Free testosterone & 0.089 & 0.337 & 0.150 & 0.173 & 0.161 & 0.158 & 0.163 & 0.112 \\
\hline Ovarian volume & 0.329 & 0.009 & 0.473 & 0.010 & -0.123 & 0.309 & 0.334 & 0.006 \\
\hline Follicle no. & 0.132 & 0.155 & 0.113 & 0.369 & 0.057 & 0.576 & 0.441 & $<0.001$ \\
\hline No. of menses/yr & -0.172 & 0.061 & -0.037 & 0.737 & - & - & - & - \\
\hline
\end{tabular}

HA, hyperandrogenism; OM, oligomenorrhea; PCOM, polycystic ovarian morphology; BMI, body mass index; WC, waist circumference; SHBG, sex hormone-binding globulin.

Table 4. Association of AMH with total testosterone by multiple linear regression analyses in women with polycystic ovary syndrome

\begin{tabular}{|c|c|c|c|c|c|}
\hline \multirow[t]{2}{*}{ Variable } & \multicolumn{2}{|c|}{$\begin{array}{c}\text { Unstandardized } \\
\text { coefficients }\end{array}$} & \multirow{2}{*}{$\begin{array}{c}\begin{array}{c}\text { Standardized } \\
\text { coefficients }\end{array} \\
\beta\end{array}$} & \multirow[t]{2}{*}{$p$ value } & \multirow[t]{2}{*}{ CI } \\
\hline & $\beta$ & SE & & & \\
\hline AMH-unadjusted & 1.06 & 0.21 & 0.33 & $<0.01$ & $0.64-1.47$ \\
\hline Age-adjusted & 1.06 & 0.21 & 0.33 & $<0.01$ & $0.64-1.48$ \\
\hline Age, BMI-adjusted & 1.03 & 0.21 & 0.32 & $<0.01$ & $0.61-1.45$ \\
\hline Age, BMI, menses/yr-adjusted & 1.01 & 0.21 & 0.31 & $<0.01$ & $0.59-1.43$ \\
\hline
\end{tabular}

$\mathrm{AMH}$, anti-Müllerian hormone; SE, standard error; CI, confidence interval; BMI, body mass index.

0.47 , all $p<0.05)$ but was not correlated with total or free testosterone (groups 3 and 4 ) (Table 2).

AMH level was significantly positively correlated with total testosterone $(r=0.208$ and $r=0.347)$ and ovarian volume $(r=0.329$ and $r=0.473)$ in women with PCOS, regardless of the presence of PCOM and was significantly positively correlated with ovarian volume $(r=0.334)$ and follicle number $(r=0.441)$ in regular cycling women without PCOM. AMH level was not significantly correlated with ovarian volume and follicle number in regular cycling women with PCOM (Table 3).

The multiple linear regression analyses showed that serum AMH was an independent determinant of total testosterone after adjustment for age, BMI, and the number of menses/year $(\beta=0.31, p<0.01)$ in women with PCOS (Table 4). AMH was also shown to be the strongest predictor of PCOS by multiple logistic regression analysis (odds ratio, 1.29; 95\% confidence interval [CI], 1.15 to 1.43) after controlling for age, BMI, total testosterone, and the number of menses/year (Table 5).

When we divided the subjects according to age into groups with 5-year intervals, serum AMH level did not show any significant age-dependence in women with PCOS or regular cycling women (Supplementary Figs. 1 and 2). The optimal AMH cutoff value for diagnosing PCOS was $10.0 \mathrm{ng} / \mathrm{mL}$ (71\% sensitivity, 93\% specificity), and the area under the receiver-operating characteristic curve was 0.88 (95\% CI, o.84 to 0.91)(Fig. 1).

\section{DISCUSSION}

In this present study, we compared the AMH levels among four groups according to the presence of PCOS and PCOM. Serum AMH level was the highest in group $1(\mathrm{HA}+\mathrm{OM}+\mathrm{PCOM})$ and the lowest in group 4 (control). Women with PCOS had higher serum AMH levels than did regular cycling women, regardless of the 
Table 5. Multiple logistic regression analysis for predicting the polycystic ovary syndrome

\begin{tabular}{lccc}
\hline Variable & Odds ratio & CI & p value \\
\hline AMH, ng/mL & 1.29 & $1.15-1.43$ & $<0.01$ \\
Age, yr & 1.01 & $0.90-1.13$ & 0.87 \\
Body mass index, $\mathrm{kg} / \mathrm{m}^{2}$ & 0.86 & $0.73-1.01$ & 0.06 \\
$\begin{array}{l}\text { Total testosterone, } \\
\text { nmol/L }\end{array}$ & 1.11 & $1.06-1.15$ & $<0.01$ \\
No. of menses/yr & 0.14 & $0.08-0.24$ & $<0.01$
\end{tabular}

CI, confidence interval; AMH, anti-Müllerian hormone.

presence of PCOM, and women with PCOM had higher serum AMH levels than did women without PCOM, regardless of the presence of PCOS.

AMH was positively associated with HA only in women with PCOS, not in regular cycling women regardless of the presence of PCOM. We also observed that serum $\mathrm{AMH}$ level did not differ between the five age groups (< 20,20 to 24,25 to 29,30 to 34 , and $\geq 35$ years). The AMH optimal cutoff value for PCOS diagnosis was $10.5 \mathrm{ng} / \mathrm{mL}$ in young Korean women.

The association of AMH level with HA observed in our study was consistent with the finding of previous studies that AMH level was positively correlated with symptom severity in PCOS $[19,20]$. AMH concentration was previously shown to be positively correlated with testosterone level in normogonadotropic anovulatory infertile women [21]. Additionally, one study showed that $\mathrm{AMH}$ level was positively related to the number of 2- to 5 -mm follicles and was positively correlated with serum testosterone in women with PCOS [6,22-24]. In our study, AMH level was significantly associated with total testosterone, even after adjustment for age, BMI, and the number of menses per year. In multiple regression analysis, the number of small follicles and serum androgen levels were positively correlated with the AMH level in women with polycystic ovaries with and without HA and in normal controls during controlled ovarian hyperstimulation. HA was associated with an additional increase in AMH level in women with polycystic ovaries [25]. HA may affect $\mathrm{AMH}$ secretion. It has been hypothesized that intra-ovarian HA promotes early follicular growth in a mechanism involving the androgen receptor [26]. Increased serum AMH level in women with PCOS could be due to increased androgen levels in the presence of an excess of small antral follicles.

The serum AMH level was significantly positively correlated with ovarian volume and follicle number in young Korean women with PCOS. AMH is produced by the granulosa cells of follicles from the time of follicle growth initiation [27]. AMH serum level has been reported to be closely correlated with small antral follicle number in both healthy women and women with PCOS $[23,28]$. Disrupted folliculogenesis resulting in the arrest of follicular growth and excess accumulation of small antral follicles may increase serum AMH level in women with PCOS [29].

Serum AMH level has been reported to decline with age [27]. However, serum AMH level was not correlated with age in women with PCOS in our study, which is consistent with the finding of no significant difference in AMH level according to age in women with PCOS in a previous study in Korean PCOS patients under the age of 40 years in which the study subjects were divided into five age groups: $\leq 20,21$ to 25,26 to 30,31 to 35 , and 36 to 40 years [30]. In a European study, serum AMH levels in women with PCOS were 2- to 3-fold higher than those in controls and remained elevated until 40 years of age [22]. The lack of AMH decline with age in women with PCOS may be due to a supraphysiologic concentration of AMH in those women compared to in regular cycling women.

The serum AMH cutoff value for PCOS diagnosis identified in our study was higher than those of previous studies. In adolescent and young adult Chinese patients (age from 17 to 25 years) with PCOS, the serum AMH cutoff value for PCOS diagnosis was $8 \mathrm{ng} / \mathrm{mL}$, with a sensitivity of $62 \%$ and a specificity of $70 \%$ [31]. In a study performed in PCOS women from a European population (age from 22 to 36.4 years), $8.40 \mathrm{ng} / \mathrm{mL}$ was suggested as the serum AMH cutoff value for PCOS diagnosis, with a sensitivity $67 \%$ and a specificity $92 \%$ [23]. ROC analysis suggested that a serum AMH value of $5 \mathrm{ng} / \mathrm{mL}$ (sensitivity $84 \%$, specificity $82 \%$ ) was the best determinant of PCOS status in Australia [32]. The difference of ethnicity could affect the different results. An ROC curve analysis showed an AMH cutoff value of $7.8 \mathrm{ng} / \mathrm{mL}$ (sensitivity $76 \%$, specificity $87 \%$ ) for PCOS diagnosis in Korean women (21 to 39 years) [33]. The differences in the AMH cutoff values determined by different studies may be due to the different methods and assays used to measure 
$\mathrm{AMH}$ and to differences in the included subjects. Compared to the previous study in Korean PCOS women [33], BMI was slightly higher in our PCOS subjects, although age did not differ between the two studies. Recently, an increase in serum AMH levels was observed in samples that had been stored for a 30-week period [34]. Because the assays used to measure AMH did not differ between these two studies, fluctuations in serum AMH level due to storage should be considered.

A strength of our study was its relatively large sample size. Because there was no difference in age between the PCOS women and regular cycling women, we could exclude the confounding effect of age on AMH levels. However, further prospective studies are needed to evaluate AMH levels in older women with PCOS (over 40 years). In a previous prospective study of PCOS women aged 35 to 39 years, there was a significant decrease in $\mathrm{AMH}$ at re-evaluation after 5 years [35]. Another limitation of this study is that because universally accepted methods and assays for measuring serum AMH level are lacking, it is difficult to generalize the results of our study.

In conclusion, we observed that serum AMH level was associated with hyperandrogenemia in women with PCOS. Serum AMH level can be useful for PCOS diagnosis at any age under 40 years. The optimal serum AMH cutoff value for PCOS diagnosis identified in this study of young Korean women was $10.0 \mathrm{ng} / \mathrm{mL}$.

\section{KEY MESSAGE}

1. The serum anti-Müllerian hormone (AMH) level can be useful for the diagnosis of polycystic ovary syndrome (PCOS) at any age less than 40 years.

2. The optimal AMH cutoff value for the diagnosis of PCOS identified in this study of young Korean women was $10.0 \mathrm{ng} / \mathrm{mL}$.

3. Serum AMH was an independent determinant of total testosterone after adjustment for age, body mass index, and the number of menses/ year.

\section{Conflict of interest}

No potential conflict of interest relevant to this article was reported.

\section{REFERENCES}

1. Azziz R, Woods KS, Reyna R, Key TJ, Knochenhauer ES, Yildiz BO. The prevalence and features of the polycystic ovary syndrome in an unselected population. J Clin Endocrinol Metab 2004;89:2745-2749.

2. Rotterdam ESHRE/ASRM-Sponsored PCOS Consensus Workshop Group. Revised 2003 consensus on diagnostic criteria and long-term health risks related to polycystic ovary syndrome. Fertil Steril 2004;81:19-25.

3. Robin G, Gallo C, Catteau-Jonard S, et al. Polycystic ovary-like abnormalities (PCO-L) in women with functional hypothalamic amenorrhea. J Clin Endocrinol Metab 2012;97:4236-4243.

4. Keevil BG. How do we measure hyperandrogenemia in patients with PCOS? J Clin Endocrinol Metab 2014;99:777779 .

5. Weenen C, Laven JS, Von Bergh AR, et al. Anti-Mullerian hormone expression pattern in the human ovary: potential implications for initial and cyclic follicle recruitment. Mol Hum Reprod 2004;10:77-83.

6. Pigny P, Merlen E, Robert Y, et al. Elevated serum level of anti-Mullerian hormone in patients with polycystic ovary syndrome: relationship to the ovarian follicle excess and to the follicular arrest. J Clin Endocrinol Metab 2003;88:5957-5962.

7. Sahmay S, Atakul N, Oncul M, Tuten A, Aydogan B, Seyisoglu H. Serum anti-Mullerian hormone levels in the main phenotypes of polycystic ovary syndrome. Eur J Obstet Gynecol Reprod Biol 2013;170:157-161.

8. Rosenfield RL, Wroblewski K, Padmanabhan V, Littlejohn E, Mortensen M, Ehrmann DA. Antimullerian hormone levels are independently related to ovarian hyperandrogenism and polycystic ovaries. Fertil Steril 2012;98:242249 .

9. Sahmay S, Aydin Y, Oncul M, Senturk LM. Diagnosis of polycystic ovary syndrome: AMH in combination with clinical symptoms. J Assist Reprod Genet 2014;31:213-220.

10. Pinola P, Morin-Papunen LC, Bloigu A, et al. Anti-Mullerian hormone: correlation with testosterone and oligo- or amenorrhoea in female adolescence in a population-based cohort study. Hum Reprod 2014;29:2317-2325.

11. Iliodromiti S, Kelsey TW, Anderson RA, Nelson SM. Can anti-Mullerian hormone predict the diagnosis of polycystic ovary syndrome? A systematic review and meta-analysis of extracted data. J Clin Endocrinol Metab 
2013;98:3332-3340.

12. Dewailly D, Gronier H, Poncelet E, et al. Diagnosis of polycystic ovary syndrome (PCOS): revisiting the threshold values of follicle count on ultrasound and of the serum AMH level for the definition of polycystic ovaries. Hum Reprod 2011;26:3123-3129.

13. Li Y, Ma Y, Chen X, et al. Different diagnostic power of anti-Mullerian hormone in evaluating women with polycystic ovaries with and without hyperandrogenism. J Assist Reprod Genet 2012;29:1147-1151.

14. Cui Y, Shi Y, Cui L, Han T, Gao X, Chen ZJ. Age-specific serum antimullerian hormone levels in women with and without polycystic ovary syndrome. Fertil Steril 2014;102:230-236.

15. Lauritsen MP, Bentzen JG, Pinborg A, et al. The prevalence of polycystic ovary syndrome in a normal population according to the Rotterdam criteria versus revised criteria including anti-Mullerian hormone. Hum Reprod 2014;29:791-801.

16. Escobar-Morreale HF, Carmina E, Dewailly D, et al. Epidemiology, diagnosis and management of hirsutism: a consensus statement by the Androgen Excess and Polycystic Ovary Syndrome Society. Hum Reprod Update 2012;18:146-170.

17. Sung YA. Hyperandrogenism in women: polycystic ovary syndrome. Hanyang Med Rev 2012;32:197-202.

18. Balen AH, Laven JS, Tan SL, Dewailly D. Ultrasound assessment of the polycystic ovary: international consensus definitions. Hum Reprod Update 2003;9:505-514.

19. Homburg R, Ray A, Bhide P, et al. The relationship of serum anti-Mullerian hormone with polycystic ovarian morphology and polycystic ovary syndrome: a prospective cohort study. Hum Reprod 2013;28:1077-1083.

20. Koninger A, Koch L, Edimiris P, et al. Anti-Mullerian hormone: an indicator for the severity of polycystic ovarian syndrome. Arch Gynecol Obstet 2014;290:1023-1030.

21. Laven JS, Mulders AG, Visser JA, Themmen AP, De Jong FH, Fauser BC. Anti-Mullerian hormone serum concentrations in normoovulatory and anovulatory women of reproductive age. J Clin Endocrinol Metab 2004;89:318323.

22. Piltonen T, Morin-Papunen L, Koivunen R, Perheentupa A, Ruokonen A, Tapanainen JS. Serum anti-Mullerian hormone levels remain high until late reproductive age and decrease during metformin therapy in women with polycystic ovary syndrome. Hum Reprod 2005;20:1820-
1826.

23. Pigny P, Jonard S, Robert Y, Dewailly D. Serum anti-Mullerian hormone as a surrogate for antral follicle count for definition of the polycystic ovary syndrome. J Clin Endocrinol Metab 2006;91:941-945.

24. Nardo LG, Yates AP, Roberts SA, Pemberton P, Laing I. The relationships between $\mathrm{AMH}$, androgens, insulin resistance and basal ovarian follicular status in non-obese subfertile women with and without polycystic ovary syndrome. Hum Reprod 2009;24:2917-2923.

25. Eldar-Geva T, Margalioth EJ, Gal M, et al. Serum anti-Mullerian hormone levels during controlled ovarian hyperstimulation in women with polycystic ovaries with and without hyperandrogenism. Hum Reprod 2005;20:1814-1819.

26. Jonard S, Dewailly D. The follicular excess in polycystic ovaries, due to intra-ovarian hyperandrogenism, may be the main culprit for the follicular arrest. Hum Reprod Update 2004;10:107-117.

27. Durlinger AL, Visser JA, Themmen AP. Regulation of ovarian function: the role of anti-Mullerian hormone. Reproduction 2002;124:601-609.

28. Chen MJ, Yang WS, Chen CL, Wu MY, Yang YS, Ho HN. The relationship between anti-Mullerian hormone, androgen and insulin resistance on the number of antral follicles in women with polycystic ovary syndrome. Hum Reprod 2008;23:952-957.

29. Wang JG, Nakhuda GS, Guarnaccia MM, Sauer MV, Lobo RA. Mullerian inhibiting substance and disrupted folliculogenesis in polycystic ovary syndrome. Am J Obstet Gynecol 2007;196:77.

30. Hwang YI, Sung NY, Koo HS, et al. Can high serum anti-Mullerian hormone levels predict the phenotypes of polycystic ovary syndrome (PCOS) and metabolic disturbances in PCOS patients? Clin Exp Reprod Med 2013;40:135-140.

31. Li L, Chen X, Mo Y, Chen Y, Wenig M, Yang D. Elevated serum anti-mullerian hormone in adolescent and young adult Chinese patients with polycystic ovary syndrome. Wien Klin Wochenschr 2010;122:519-524.

32. Tremellen K, Zander-Fox D. Serum anti-Mullerian hormone assessment of ovarian reserve and polycystic ovary syndrome status over the reproductive lifespan. Aust N Z J Obstet Gynaecol 2015;55:384-389.

33. Woo HY, Kim KH, Rhee EJ, Park H, Lee MK. Differences of the association of anti-Mullerian hormone with 
clinical or biochemical characteristics between women with and without polycystic ovary syndrome. Endocr J 2012;59:781-790.

34. Han X, McShane M, Sahertian R, White C, Ledger W. Pre-mixing serum samples with assay buffer is a prerequisite for reproducible anti-Mullerian hormone mea- surement using the Beckman Coulter Gen II assay. Hum Reprod 2014;29:1042-1048.

35. Carmina E, Campagna AM, Mansuet P, Vitale G, Kort D, Lobo R. Does the level of serum antimullerian hormone predict ovulatory function in women with polycystic ovary syndrome with aging? Fertil Steril 2012;98:1043-1046. 


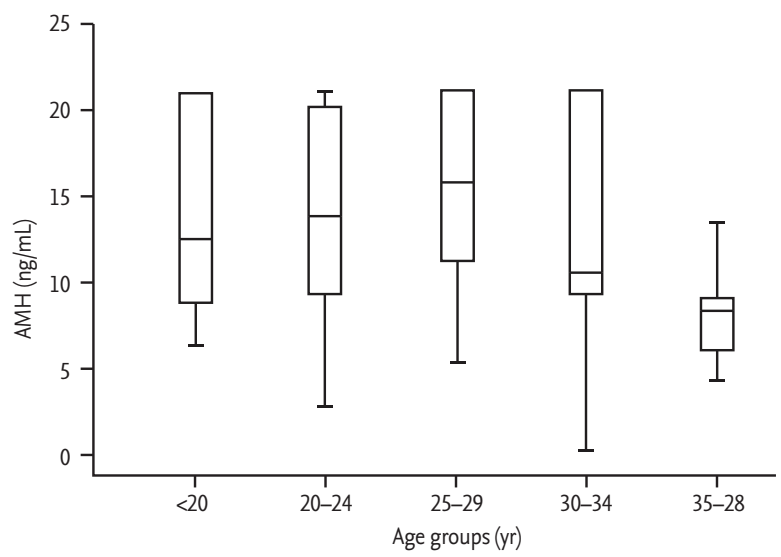

\begin{tabular}{cc}
\hline Age group, yr & Women with PCOS $(n=207)$ \\
\hline$<20(n=25)$ & $12.3(8.5-21.0)$ \\
$20-24(n=85)$ & $13.6(9.1-20.2)$ \\
$25-29(n=69)$ & $15.7(10.9-21.0)^{\mathrm{a}}$ \\
$30-34(n=21)$ & $10.5(8.9-21.0)$ \\
$35-38(n=7)$ & $8.0(5.5-10.0)$ \\
\hline
\end{tabular}

Supplementary Figure 1. Serum anti-Müllerian hormone (AMH) levels according to age groups in women with polycystic ovary syndrome (PCOS). Values are presented as median (interquartile range). $p$ for trend was 0.048 . ${ }^{a} p<0.05$ vs. age group with 35 to 38 years.

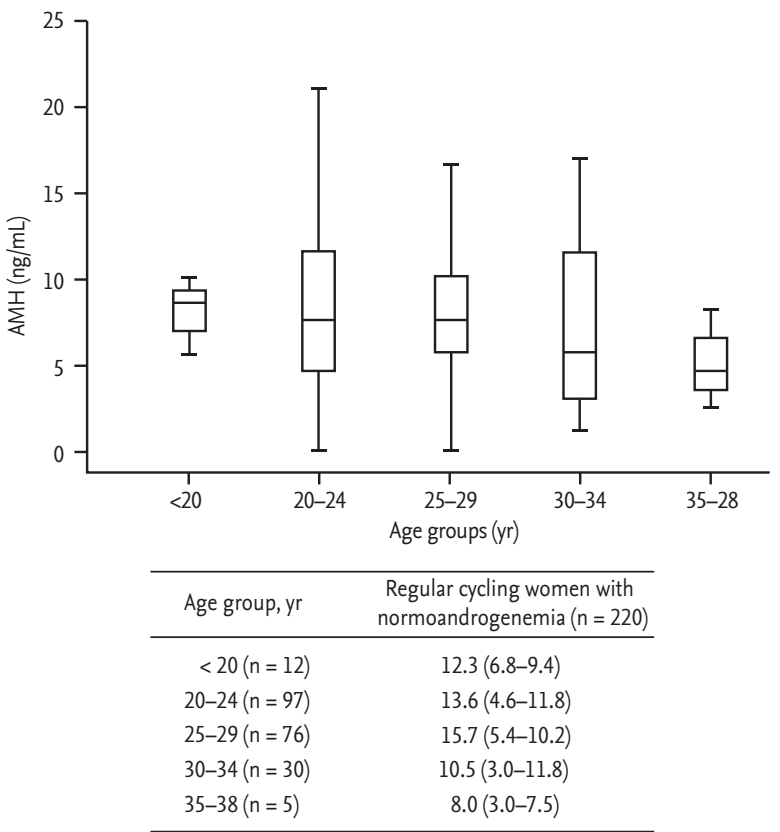

Supplementary Figure 2. Serum anti-Müllerian hormone (AMH) levels according to age groups in regular cycling women with normoandrogenemia. Values are presented as median (interquartile range). $p$ for trend was 0.333 . 